\title{
Local Fractional Poisson and Laplace Equations with Applications to Electrostatics in Fractal Domain
}

\author{
Yang-Yang Li, ${ }^{1,2}$ Yang Zhao, ${ }^{3}$ Gong-Nan Xie, ${ }^{4}$ Dumitru Baleanu, ${ }^{5,6,7}$ \\ Xiao-Jun Yang, ${ }^{8}$ and Kai Zhao' \\ ${ }^{1}$ Northeast Institute of Geography and Agroecology, Chinese Academy of Sciences, Changchun 130102, China \\ ${ }^{2}$ University of Chinese Academy of Sciences, Beijing 100049, China \\ ${ }^{3}$ Electronic and Information Technology Department, Jiangmen Polytechnic, Jiangmen 529090, China \\ ${ }^{4}$ School of Mechanical Engineering, Northwestern Polytechnical University, Xian, Shaanxi 710048, China \\ ${ }^{5}$ Department of Chemical and Materials Engineering, Faculty of Engineering, King Abdulaziz University, P.O. Box 80204, \\ Jeddah 21589, Saudi Arabia \\ ${ }^{6}$ Department of Mathematics and Computer Sciences, Faculty of Arts and Sciences, Cankaya University, 06530 Ankara, Turkey \\ ${ }^{7}$ Institute of Space Sciences, Magurele, 077125 Bucharest, Romania \\ ${ }^{8}$ Department of Mathematics and Mechanics, China University of Mining and Technology, Xuzhou, Jiangsu 221008, China
}

Correspondence should be addressed to Kai Zhao; dzhaokai@163.com

Received 19 March 2014; Accepted 1 April 2014; Published 16 April 2014

Academic Editor: Carlo Cattani

Copyright (C) 2014 Yang-Yang Li et al. This is an open access article distributed under the Creative Commons Attribution License, which permits unrestricted use, distribution, and reproduction in any medium, provided the original work is properly cited.

From the local fractional calculus viewpoint, Poisson and Laplace equations were presented in this paper. Their applications to the electrostatics in fractal media are discussed and their local forms in the Cantor-type cylindrical coordinates are also obtained.

\section{Introduction}

Poisson and Laplace equations had successfully played an important role in electrodynamics [1-3]. Mathematically, they are two-order partial differential equations and exist in the spaces of different kinds $[4,5]$. Their solutions were studied by different. There are approximate and numerical methods for them, such as the finite difference method [6], the finite element method [7], the random walk method [8], the quadrilateral quadrature element [9], and the complex polynomial method [10].

Since Mandelbrot [10] described the fractals, the fractional calculus [11-13] and local fractional calculus [14-16] were applied to the real world problem based on them. For example, Engheta discussed the fractional-order electromagnetic theory [17]. Tarasov studied the fractal distribution of charges [18]. Calcagni et al. suggested the electric charge in multiscale space and times [19]. In [20], the local fractional approach for Maxwell's equations was considered. Local fractional calculus [20-25] has been successfully applied to describe dynamical systems with the nondifferentiable functions. For example, the Maxwell theory on Cantor sets was studied in [20]. The Heisenberg uncertainty relation was discussed by using the local fractional Fourier analysis [21]. The system of Navier-Stokes equations arising in fractal flows was reported in [22]. The local fractional nonhomogeneous heat equations arising in fractal heat flow were presented in [23]. The fractal forest gap within the local fractional derivative was investigated in [24].

In the present paper, the local fractional Poisson and Laplace equations within the nondifferentiable functions arising in electrostatics in fractal domain and in the Cantortype cylindrical coordinates [25] based upon the local fractional Maxwell equations [20] will be derived from the fractional vector calculus.

The outline of the paper is depicted below. Section 2 introduces the local fractional Maxwell equations. Section 3 discusses the local fractional Poisson and Laplace equations 
arising in electrostatics in fractal media. In Section 4, the local fractional Poisson and Laplace equations in the Cantortype cylindrical coordinates are presented. Finally, Section 5 is devoted to the conclusions.

\section{Local Fractional Maxwell's Equations}

In this section, the local fractional Maxwell's equations are introduced and the concepts of the local fractional vector calculus are reviewed. We first introduce the local fractional vector calculus and its theorems [14, 20-23].

The local fractional line integral of the function $\mathbf{u}$ along a fractal line $l^{\alpha}$ was defined as $[14,20]$

$$
\int_{l^{(\alpha)}} \mathbf{u}\left(x_{P}, y_{P}, z_{P}\right) \cdot d \mathbf{l}^{(\alpha)}=\lim _{N \rightarrow \infty} \sum_{P=1}^{N} \mathbf{u}\left(x_{P}, y_{P}, z_{P}\right) \cdot \Delta \mathbf{l}_{P}^{(\alpha)},
$$

where the quantity $\Delta \mathbf{l}_{P}^{(\alpha)}$ is elements of line, $\left|\Delta \mathbf{l}_{P}^{(\alpha)}\right| \rightarrow 0$ as $N \rightarrow \infty$, and $\alpha \in(0,1]$.

The local fractional surface integral was defined as $[14$, 20-23]

$$
\iint \mathbf{u}\left(r_{P}\right) \cdot d \mathbf{S}^{(\beta)}=\lim _{N \rightarrow \infty} \sum_{P=1}^{N} \mathbf{u}\left(r_{P}\right) \cdot \mathbf{n}_{P} \Delta S_{P}^{(\beta)},
$$

where the quantity $\Delta S_{P}^{(\beta)}$ is elements of surface, the quantity $\mathbf{n}_{P}$ is $N$ elements of area with a unit normal local fractional vector, and $\Delta S_{P}^{(\beta)} \rightarrow 0$ as $N \rightarrow \infty$ for $\beta=2 \alpha$.

The local fractional volume integral of the function $\mathbf{u}$ was defined as $[14,20-23]$

$$
\iiint \mathbf{u}\left(r_{P}\right) d V^{(\gamma)}=\lim _{N \rightarrow \infty} \sum_{P=1}^{N} \mathbf{u}\left(r_{P}\right) \Delta V_{P}^{(\gamma)},
$$

where the quantity $\Delta V_{P}^{(\gamma)}$ is the elements of volume, $\Delta V_{P}^{(\gamma)} \rightarrow$ 0 as $N \rightarrow \infty$, and $\gamma=3 \alpha$.

The local fractional Stokes' theorem of the fractal field states that $[13,20]$

$$
\oint_{l^{(\alpha)}} \mathbf{u} \cdot d \mathbf{l}^{(\alpha)}=\iint_{S^{(\beta)}}\left(\nabla^{\alpha} \times \mathbf{u}\right) \cdot d \mathbf{S}^{(\beta)} .
$$

The electric Gauss law for the fractal electric field was suggested as [20]

$$
\oiint_{S^{(\beta)}} D \cdot d \mathbf{S}^{(\beta)}=\iiint_{V^{(\gamma)}} \rho d V^{(\gamma)},
$$

which leads to

$$
\nabla^{\alpha} \cdot D=\rho,
$$

where the quantity $\rho$ denotes the free charges density and the quantity $D$ is the fractal electric displacement.

The Ampere law in the fractal magnetic field was presented as [20]

$$
\oint_{l^{(\alpha)}} H \cdot d \mathbf{l}^{(\alpha)}=\iint_{S^{(\beta)}}\left(J_{a}+\frac{\partial^{\alpha} D}{\partial t^{\alpha}}\right) \cdot d \mathbf{S}^{(\beta)},
$$

which leads to

$$
\nabla^{\alpha} \times H=J_{a}+\frac{\partial^{\alpha} D}{\partial t^{\alpha}}
$$

where the quantity $H$ is the fractal magnetic field strength and the quantity $J_{a}$ denotes the fractal conductive current.

The Faraday law in the fractal electric field reads as [20]

$$
\oint_{l^{(\alpha)}} E \cdot d \mathbf{l}^{(\alpha)}+\frac{\partial^{\alpha}}{\partial t^{\alpha}} \iint_{S^{(\beta)}} B \cdot d \mathbf{S}^{(\beta)}=0,
$$

which leads to

$$
\nabla^{\alpha} \times E=-\frac{\partial^{\alpha} B}{\partial t^{\alpha}}
$$

where the constitutive relationships in fractal electric field are

$$
D=\varepsilon_{f} E
$$

with the fractal dielectric permittivity $\varepsilon_{f}$ and the fractal dielectric field $E$.

The magnetic Gauss law for the fractal magnetic field was written as [20]

$$
\oiint_{S^{(\beta)}} B \cdot d \mathbf{S}^{(\beta)}=0
$$

which leads to

$$
\nabla^{\alpha} \cdot B=0
$$

where the constitutive relationships in fractal magnetic field are

$$
H=\mu_{f} B
$$

with the fractal magnetic permeability $\mu_{f}$ and the fractal magnetic field $B$.

\section{Local Fractional Poisson and Laplace Equations in Fractal Media}

In this section, we derive the local fractional Poisson and Laplace equations arising in electrostatics in fractal media.

In view of (11), from (6) we have

$$
\nabla^{\alpha} \cdot\left(\varepsilon_{f} E\right)=\rho
$$

so that

$$
\varepsilon_{f}\left(\nabla^{\alpha} \cdot E\right)=\rho,
$$

where $\rho$ denotes the free charges density in fractal homogeneous medium, $\varepsilon_{f}$ denotes the fractal dielectric permittivity, and $E$ denotes the fractal dielectric field.

Hence, the local fractional differential form of Gauss's law in local fractional divergence operator reads as

$$
\nabla^{\alpha} \cdot E=\frac{\rho}{\varepsilon_{f}} .
$$


If the electrostatics in fractal domain is described by the expression

$$
\nabla^{\alpha} \times E=-\frac{\partial^{\alpha} B}{\partial t^{\alpha}}=0
$$

then the fractal electric field within the local fractional gradient is

$$
E=-\nabla^{\alpha} \psi
$$

where the quantity $\psi$ is a nondifferentiable term and $E$ is the fractal dielectric field.

In view of (16) and (19), we obtain

$$
\varepsilon_{f}\left[\nabla^{\alpha} \cdot\left(-\nabla^{\alpha} \psi\right)\right]=\rho,
$$

which leads to

$$
-\varepsilon_{f}\left[\nabla^{\alpha} \cdot \nabla^{\alpha} \psi\right]=\rho
$$

where the quantity $\psi$ is a nondifferentiable term and $\varepsilon_{f}$ denotes the fractal dielectric permittivity.

From (21) we arrive at

$$
\nabla^{\alpha} \cdot \nabla^{\alpha} \psi=-\frac{\rho}{\varepsilon_{f}} .
$$

Let us define the local fractional operator

$$
\nabla^{\alpha} \cdot \nabla^{\alpha}=\nabla^{2 \alpha}
$$

Making use of (22) and (23), we have

$$
\nabla^{2 \alpha} \psi=-\frac{\rho}{\varepsilon_{f}}
$$

In the Cantorian coordinates, from (24), the local fractional Poisson equation arising in electrostatics in fractal domain can be written as

$$
\begin{aligned}
& \frac{\partial^{2 \alpha}}{\partial x^{2 \alpha}} \psi(x, y, z)+\frac{\partial^{2 \alpha}}{\partial y^{2 \alpha}} \psi(x, y, z)+\frac{\partial^{2 \alpha}}{\partial z^{2 \alpha}} \psi(x, y, z) \\
& \quad=-\frac{\rho(x, y, z)}{\varepsilon_{f}},
\end{aligned}
$$

where both $\psi(x, y, z)$ and $\rho(x, y, z)$ are nondifferentiable functions; the local fractional operator $\nabla^{2 \alpha}$ in the Cantorian coordinates was written as [14]

$$
\nabla^{2 \alpha}=\frac{\partial^{2 \alpha}}{\partial x^{2 \alpha}}+\frac{\partial^{2 \alpha}}{\partial y^{2 \alpha}}+\frac{\partial^{2 \alpha}}{\partial z^{2 \alpha}}
$$

In the Cantorian coordinates, from (25), the local fractional Laplace equation arising in electrostatics in fractal domain is

$$
\frac{\partial^{2 \alpha}}{\partial x^{2 \alpha}} \psi(x, y, z)+\frac{\partial^{2 \alpha}}{\partial y^{2 \alpha}} \psi(x, y, z)+\frac{\partial^{2 \alpha}}{\partial z^{2 \alpha}} \psi(x, y, z)=0,
$$

where the quantity $\psi(x, y, z)$ is a nondifferentiable function.

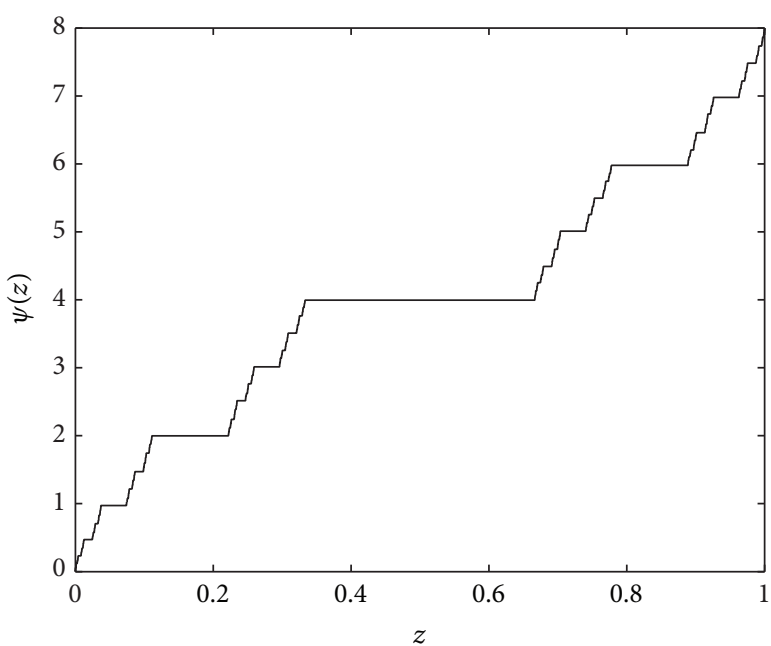

Figure 1: Plot of (31) with parameters $\alpha=\ln 2 / \ln 3$ and $b=1$.

From (25) the local fractional Laplace equation arising in electrostatics in fractal domain with two variables can be written as

$$
\frac{\partial^{2 \alpha}}{\partial x^{2 \alpha}} \psi(x, y)+\frac{\partial^{2 \alpha}}{\partial y^{2 \alpha}} \psi(x, y)=-\frac{\rho(x, y)}{\varepsilon_{f}},
$$

where both $\psi(x, y)$ and $\rho(x, y)$ are nondifferentiable functions.

From (27) the local fractional Laplace equation arising in electrostatics in fractal domain with two variables can be written as

$$
\frac{\partial^{2 \alpha}}{\partial x^{2 \alpha}} \psi(x, y)+\frac{\partial^{2 \alpha}}{\partial y^{2 \alpha}} \psi(x, y)=0
$$

where the quantity $\psi(x, y)$ is a nondifferentiable function.

For the boundary conditions on the fractal potential

$$
\psi(0)=0, \quad \psi(b)=8
$$

we have the local fractional Laplace's equation

$$
\frac{\partial^{2 \alpha}}{\partial z^{2 \alpha}} \psi(z)=0
$$

which leads to the nondifferentiable solution given $y$

$$
\psi(z)=8\left(\frac{z}{b}\right)^{\alpha}
$$

and its graph is shown in Figure 1.

We notice that the local fractional Poisson's equation shows the potential behavior in the fractal regions with nondifferentiable functions where there is the free charge, while local fractional Laplace's equation governs the nondifferentiable potential behavior in fractal regions where there is no free charge. 


\section{Local Fractional Poisson and Laplace Equations in the Cantor-Type Cylindrical Coordinates}

In this section, the local fractional Poisson and Laplace equations in the Cantor-type cylindrical coordinates are considered. We first start with the Cantor-type cylindrical coordinate method.

We now consider the Cantor-type cylindrical coordinates given by $[14,25]$

$$
\begin{gathered}
x^{\alpha}=R^{\alpha} \cos _{\alpha} \theta^{\alpha}, \\
y^{\alpha}=R^{\alpha} \sin _{\alpha} \theta^{\alpha}, \\
z^{\alpha}=z^{\alpha}
\end{gathered}
$$

with $R \in(0,+\infty), z \in(-\infty,+\infty), \theta \in(0, \pi]$, and $x^{2 \alpha}+y^{2 \alpha}=$ $R^{2 \alpha}$.

From (33) we have [25]

$$
\begin{gathered}
\nabla^{\alpha} \phi(R, \theta, z)=\mathbf{e}_{R}^{\alpha} \frac{\partial^{\alpha}}{\partial R^{\alpha}} \phi+\mathbf{e}_{\theta}^{\alpha} \frac{1}{R^{\alpha}} \frac{\partial^{\alpha}}{\partial \theta^{\alpha}} \phi+\mathbf{e}_{z}^{\alpha} \frac{\partial^{\alpha}}{\partial z^{\alpha}} \phi, \\
\nabla^{2 \alpha} \phi(R, \theta, z)=\frac{\partial^{2 \alpha}}{\partial R^{2 \alpha}} \phi+\frac{1}{R^{2 \alpha}} \frac{\partial^{2 \alpha}}{\partial \theta^{2 \alpha}} \phi+\frac{1}{R^{\alpha}} \frac{\partial^{\alpha}}{\partial R^{\alpha}} \phi+\frac{\partial^{2 \alpha}}{\partial z^{2 \alpha}} \phi,
\end{gathered}
$$

where

$$
\begin{gathered}
\mathbf{e}_{R}^{\alpha}=\cos _{\alpha} \theta^{\alpha} \mathbf{e}_{1}^{\alpha}+\sin _{\alpha} \theta^{\alpha} \mathbf{e}_{2}^{\alpha}, \\
\mathbf{e}_{\theta}^{\alpha}=-\sin _{\alpha} \theta^{\alpha} \mathbf{e}_{1}^{\alpha}+\cos _{\alpha} \theta^{\alpha} \mathbf{e}_{2}^{\alpha}, \\
\mathbf{e}_{z}^{\alpha}=\mathbf{e}_{3}^{\alpha},
\end{gathered}
$$

and the local fractional vector suggested by

$$
\mathbf{r}=R^{\alpha} \cos _{\alpha} \theta^{\alpha} \mathbf{e}_{1}^{\alpha}+R^{\alpha} \sin _{\alpha} \theta^{\alpha} \mathbf{e}_{2}^{\alpha}+z^{\alpha} \mathbf{e}_{3}^{\alpha}=r_{R} \mathbf{e}_{R}^{\alpha}+r_{\theta} \mathbf{e}_{\theta}^{\alpha}+r_{z} \mathbf{e}_{z}^{\alpha} .
$$

In view of (35), the local fractional Poisson equation in the Cantor-type cylindrical coordinates is written as

$$
\begin{aligned}
& \frac{\partial^{2 \alpha}}{\partial R^{2 \alpha}} \psi(R, \theta, z)+\frac{1}{R^{2 \alpha}} \frac{\partial^{2 \alpha}}{\partial \theta^{2 \alpha}} \psi(R, \theta, z)+\frac{1}{R^{\alpha}} \frac{\partial^{\alpha}}{\partial R^{\alpha}} \psi(R, \theta, z) \\
& \quad+\frac{\partial^{2 \alpha}}{\partial z^{2 \alpha}} \psi(R, \theta, z)=-\frac{1}{\varepsilon_{f}} \rho(R, \theta, z),
\end{aligned}
$$

where both $\psi(R, \theta, z)$ and $\rho(R, \theta, z)$ are nondifferentiable functions.

From (35), the local fractional Laplace equation in the Cantor-type cylindrical coordinates is

$$
\begin{aligned}
\frac{\partial^{2 \alpha}}{\partial R^{2 \alpha}} & \psi(R, \theta, z)+\frac{1}{R^{2 \alpha}} \frac{\partial^{2 \alpha}}{\partial \theta^{2 \alpha}} \psi(R, \theta, z) \\
& +\frac{1}{R^{\alpha}} \frac{\partial^{\alpha}}{\partial R^{\alpha}} \psi(R, \theta, z)+\frac{\partial^{2 \alpha}}{\partial z^{2 \alpha}} \psi(R, \theta, z)=0,
\end{aligned}
$$

where the quantity $\psi(R, \theta, z)$ is a nondifferentiable function.
We now consider the Cantor-type circle coordinates given by [14]

$$
\begin{aligned}
& x^{\alpha}=R^{\alpha} \cos _{\alpha} \theta^{\alpha}, \\
& y^{\alpha}=R^{\alpha} \sin _{\alpha} \theta^{\alpha}
\end{aligned}
$$

with $R \in(0,+\infty), \theta \in(0,2 \pi]$, and $x^{2 \alpha}+y^{2 \alpha}=R^{2 \alpha}$.

Making use of (37), we obtain

$$
\begin{gathered}
\nabla^{\alpha} \phi(R, \theta)=\mathbf{e}_{R}^{\alpha} \frac{\partial^{\alpha}}{\partial R^{\alpha}} \phi+\mathbf{e}_{\theta}^{\alpha} \frac{1}{R^{\alpha}} \frac{\partial^{\alpha}}{\partial \theta^{\alpha}} \phi, \\
\nabla^{2 \alpha} \phi(R, \theta)=\frac{\partial^{2 \alpha}}{\partial R^{2 \alpha}} \phi+\frac{1}{R^{2 \alpha}} \frac{\partial^{2 \alpha}}{\partial \theta^{2 \alpha}} \phi+\frac{1}{R^{\alpha}} \frac{\partial^{\alpha}}{\partial R^{\alpha}} \phi,
\end{gathered}
$$

where [14]

$$
\begin{aligned}
& \mathbf{e}_{R}^{\alpha}=\cos _{\alpha} \theta^{\alpha} \mathbf{e}_{1}^{\alpha}+\sin _{\alpha} \theta^{\alpha} \mathbf{e}_{2}^{\alpha}, \\
& \mathbf{e}_{\theta}^{\alpha}=-\sin _{\alpha} \theta^{\alpha} \mathbf{e}_{1}^{\alpha}+\cos _{\alpha} \theta^{\alpha} \mathbf{e}_{2}^{\alpha},
\end{aligned}
$$

and the local fractional vector is suggested by [14]

$$
\mathbf{r}=R^{\alpha} \cos _{\alpha} \theta^{\alpha} \mathbf{e}_{1}^{\alpha}+R^{\alpha} \sin _{\alpha} \theta^{\alpha} \mathbf{e}_{2}^{\alpha}=r_{R} \mathbf{e}_{R}^{\alpha}+r_{\theta} \mathbf{e}_{\theta}^{\alpha} .
$$

From (28) and (42) the local fractional Poisson equation in fractal domain with two variables can be written as

$$
\begin{aligned}
& \frac{\partial^{2 \alpha}}{\partial R^{2 \alpha}} \psi(R, \theta)+\frac{1}{R^{2 \alpha}} \frac{\partial^{2 \alpha}}{\partial \theta^{2 \alpha}} \psi(R, \theta)+\frac{1}{R^{\alpha}} \frac{\partial^{\alpha}}{\partial R^{\alpha}} \psi(R, \theta) \\
& \quad=-\frac{1}{\varepsilon_{f}} \rho(R, \theta),
\end{aligned}
$$

where both $\psi(R, \theta)$ and $\rho(R, \theta)$ are nondifferentiable functions.

From (29) and (42) the local fractional Laplace equation in fractal domain with two variables can be written as

$$
\frac{\partial^{2 \alpha}}{\partial R^{2 \alpha}} \psi(R, \theta)+\frac{1}{R^{2 \alpha}} \frac{\partial^{2 \alpha}}{\partial \theta^{2 \alpha}} \psi(R, \theta)+\frac{1}{R^{\alpha}} \frac{\partial^{\alpha}}{\partial R^{\alpha}} \psi(R, \theta)=0,
$$

where the quantity $\psi(R, \theta)$ is a nondifferentiable function.

\section{Conclusions}

In this work we derived the local fractional Poisson and Laplace equations arising in electrostatics in fractal domain from local fractional vector calculus. The local fractional Poisson and Laplace equations in the Cantor-type cylindrical coordinates were also discussed. The nondifferentiable solution for local fractional Laplace equation was also given.

\section{Conflict of Interests}

The authors declare that they have no conflict of interests regarding this paper.

\section{Acknowledgment}

This work was supported by the National Natural Science Foundation of China (no. 41371345 and no. 41201335). 


\section{References}

[1] D. J. Griffiths and C. Reed, Introduction to Electrodynamics, Prentice Hall, Upper Saddle River, NJ, USA, 1999.

[2] M. N. Sadiku, Numerical Techniques in Electromagnetic, CRC Press, 2000.

[3] D. Gilbarg and N. S. Trudinger, Elliptic Partial Differential Equations of Second Order, Springer, Berlin, Germany, 2001.

[4] E. M. Poliščuk, "Equations of the Laplace and Poisson type in a function space," Matematicheskii Sbornik, vol. 114, no. 2, pp. 261-292, 1967.

[5] S. Persides, "The Laplace and Poisson equations in Schwarzschild's space-time," Journal of Mathematical Analysis and Applications, vol. 43, pp. 571-578, 1973.

[6] J. Eve and H. I. Scoins, "A note on the approximate solution of the equations of Poisson and Laplace by finite difference methods," The Quarterly Journal of Mathematics, vol. 7, pp. 217223, 1956

[7] J. Franz and M. Kasper, "Superconvergent finite element solutions of laplace and poisson equation," IEEE Transactions on Magnetics, vol. 32, no. 3, pp. 643-646, 1996.

[8] M. K. Chati, M. D. Grigoriu, S. S. Kulkarni, and S. Mukherjee, "Random walk method for the two- and three-dimensional Laplace, Poisson and Helmholtz's equations," International Journal for Numerical Methods in Engineering, vol. 51, no. 10, pp. 1133-1156, 2001.

[9] A. C. Poler, A. W. Bohannon, S. J. Schowalter, and T. V. Hromadka, II, "Using the complex polynomial method with Mathematica to model problems involving the Laplace and Poisson equations," Numerical Methods for Partial Differential Equations, vol. 25, no. 3, pp. 657-667, 2009.

[10] B. B. Mandelbrot, The Fractal Geometry of Nature, Macmillan, 1983.

[11] B. J. West, M. Bologna, and P. Grigolini, Physics of Fractal Operators, Springer, New York, NY, USA, 2003.

[12] E. Goldfain, "Fractional dynamics, Cantorian space-time and the gauge hierarchy problem," Chaos, Solitons and Fractals, vol. 22, no. 3, pp. 513-520, 2004.

[13] V. E. Tarasov, Fractional Dynamics: Applications of Fractional Calculus to Dynamics of Particles, Fields and Media, Springer, 2011.

[14] X.-J. Yang, Advanced Local Fractional Calculus and Its Applications, World Science, New York, NY, USA, 2012.

[15] J. A. T. Machado, D. Baleanu, and A. C. J. Luo, Discontinuity and Complexity in Nonlinear Physical Systems, Springer, 2014.

[16] X.-J. Yang, Local Fractional Functional Analysis and Its Applications, Asian Academic, Hong Kong, 2011.

[17] N. Engheta, "On the role of fractional calculus in electromagnetic theory," IEEE Antennas and Propagation Magazine, vol. 39, no. 4, pp. 35-46, 1997.

[18] V. E. Tarasov, "Multipole moments of fractal distribution of charges," Modern Physics Letters B, vol. 19, no. 22, pp. 1107-1118, 2005.

[19] G. Calcagni, J. Magueijo, and D. R. Fernández, "Varying electric charge in multiscale spacetimes," Physical Review D, vol. 89, no. 2, Article ID 024021, 2014.

[20] Y. Zhao, D. Baleanu, C. Cattani, D.-F. Cheng, and X.-J. Yang, "Maxwell's equations on Cantor sets: a local fractional approach," Advances in High Energy Physics, Article ID 686371, 6 pages, 2013.
[21] X.-J. Yang, D. Baleanu, and J. A. T. Machado, "Mathematical aspects of the Heisenberg uncertainty principle within local fractional Fourier analysis," Boundary Value Problems, vol. 2013, no. 1, article 131, 16 pages, 2013.

[22] X.-J. Yang, D. Baleanu, and J. A. Tenreiro Machado, "Systems of Navier-Stokes equations on Cantor sets," Mathematical Problems in Engineering, vol. 2013, Article ID 769724, 8 pages, 2013.

[23] A. M. Yang, C. Cattani, C. Zhang, G. N. Xie, and X.-J. Yang, "Local fractional Fourier series solutions for nonhomogeneous heat equations arising in fractal heat flow with local fractional derivative," Advances in Mechanical Engineering, vol. 2014, Article ID 514639, 5 pages, 2014.

[24] C.-Y. Long, Y. Zhao, and H. Jafari, "Mathematical models arising in the fractal forest gap via local fractional calculus," Abstract and Applied Analysis, vol. 2014, Article ID 782393, 6 pages, 2014.

[25] X.-J. Yang, H. M. Srivastava, J.-H. He, and D. Baleanu, "Cantortype cylindrical-coordinate method for differential equations with local fractional derivatives," Physics Letters A, vol. 377, no. 28-30, pp. 1696-1700, 2013. 


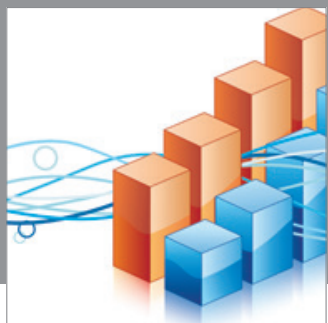

Advances in

Operations Research

mansans

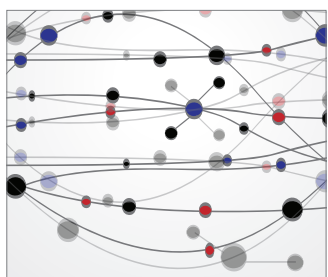

The Scientific World Journal
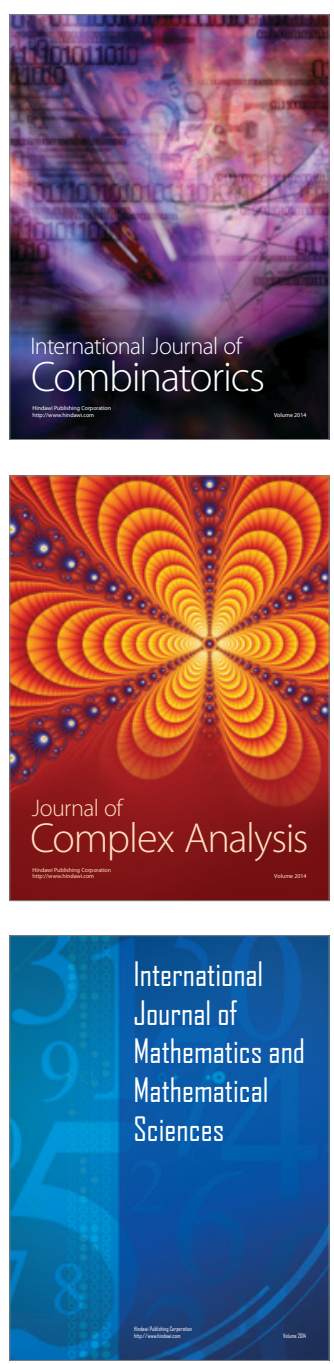
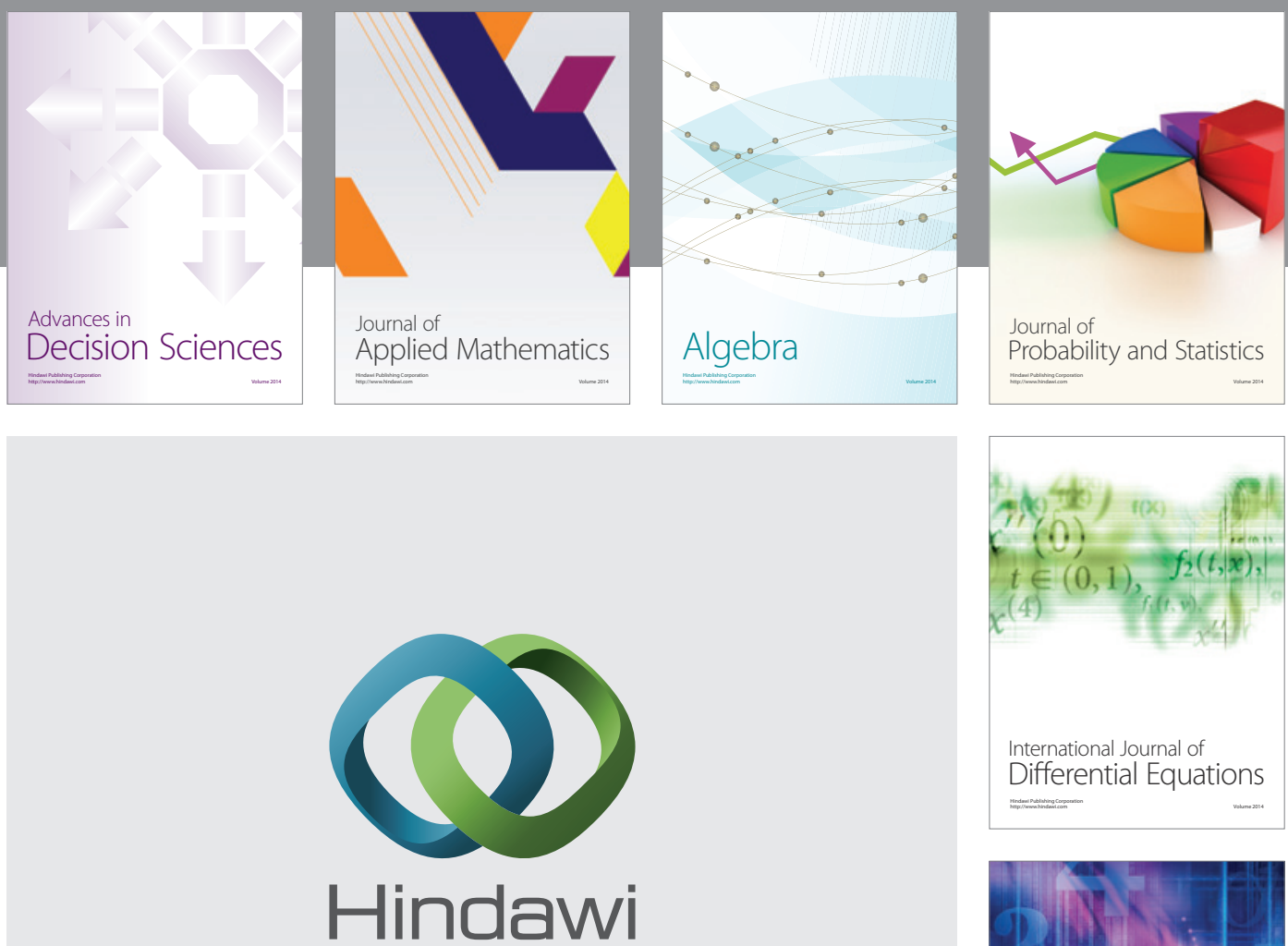

Submit your manuscripts at http://www.hindawi.com
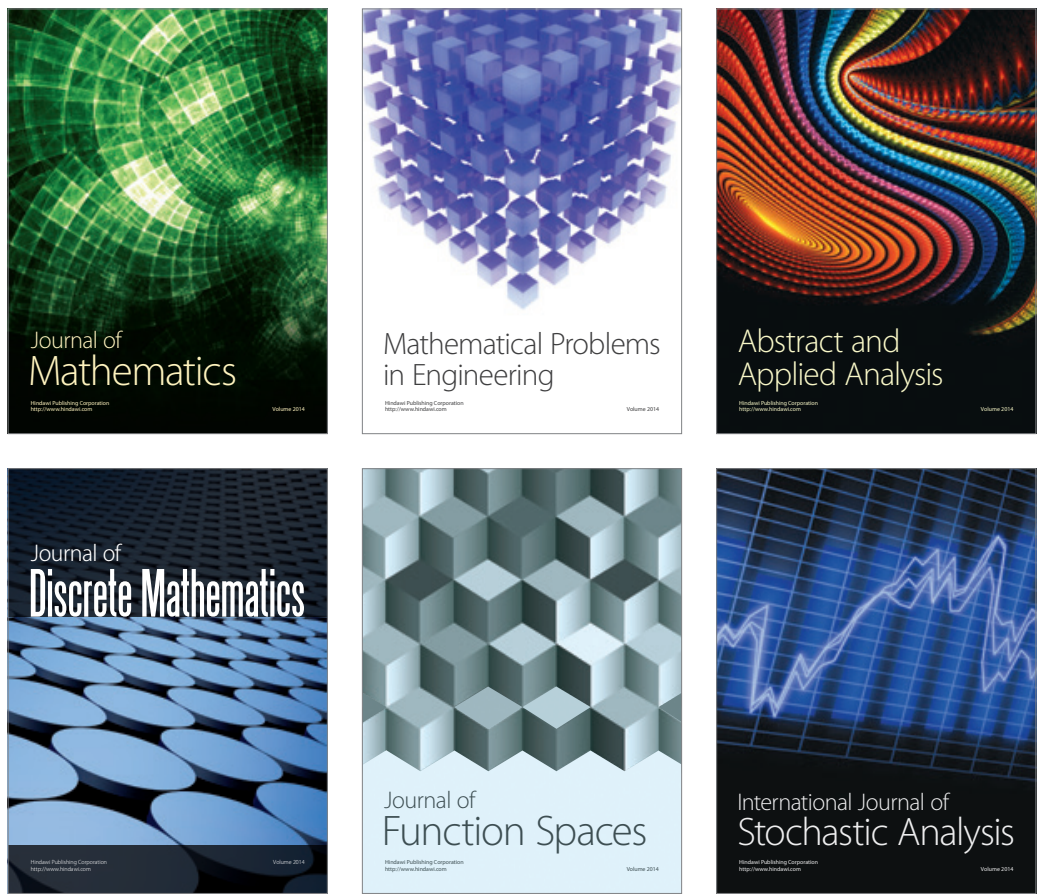

Journal of

Function Spaces

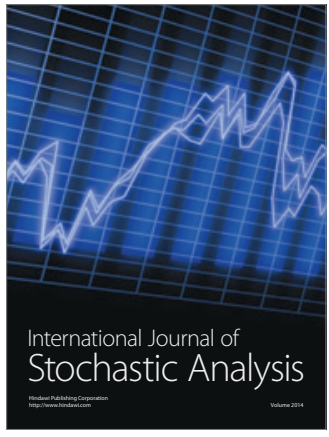

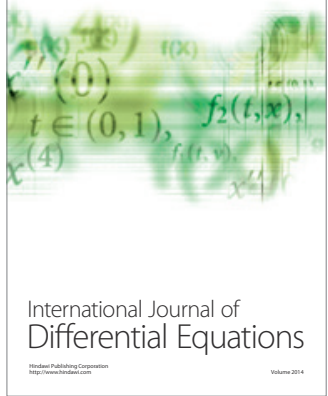
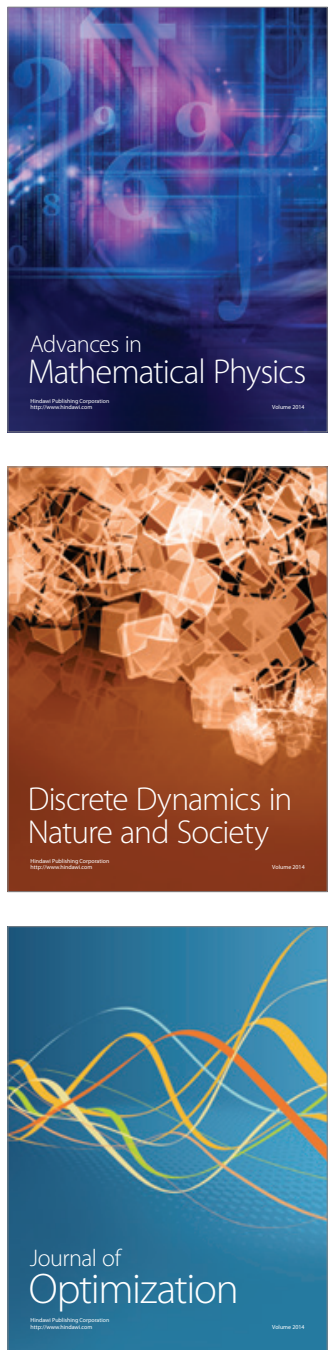